\title{
BREAST CANCER IN WOMEN: CHARACTERIZATION OF CASES INCLUDED IN THE HOSPITAL CANCER RECORDS OF THE STATE OF SÃO PAULO AND FACTORS ASSOCIATED WITH ADVANCED STADIUMS
}

Raissa Janine de Almeida' ${ }^{1}$, Arthur Felipe Decker ${ }^{1}$, Carolina Terra de Moraes Luizaga², Cristiane MurtaNascimento ${ }^{1}$

${ }^{1}$ Faculdade de Medicina de Botucatu - Botucatu (SP), Brazil. ${ }^{2}$ Fundação Oncocentro de São Paulo - São Paulo (SP), Brazil.

Introduction: Breast cancer is a worldwide public health problem, being the most common malignancy in the female population. It is a potentially curable disease if diagnosed early. The advanced stage at the time of diagnosis is associated with increased morbidity and low survival of these women. Objectives: To describe the sociodemographic, clinical, and anatomopathological characteristics of breast cancer cases in women included in the São Paulo State hospital cancer database (registros hospitalares de câncer de São Paulo - RHC-SP), established in 2000 and maintained by the Fundação Oncocentro de São Paulo (FOSP) and to investigate the factors associated with the clinical stage at the time of diagnosis. Material and methods: The study design was a series of cases. The sample consisted of women with breast cancer diagnosed between 2000-2014 and included in the RHC-FOSP. The outcome variable was the clinical stage (stage 0-II versus III-IV). The explanatory variables were: age at diagnosis and education level. This study was approved by the Human Research Ethics Committee of the Botucatu Medical School, UNESP. Results: The study included 84,987 women with in situ and invasive breast cancer diagnosed between 2000-2014. The mean age of women at diagnosis was 56.7 years (95\%CI 56.6-56.8 years). Sixty-five percent of the cases have complete elementary school or less and the most frequent histological type was ductal carcinoma (77.2\%). During the study period, there was a small decrease in the proportion of tumors in more advanced stages, from $39.8 \%$ in 2000 to $32.6 \%$ in 2014. There was a statistically significant association between the variables age at diagnosis and level of education with the clustered clinical stage of women. Women of older age and those with a higher level of education had reduced odds ratios for tumors in more advanced stages at the time of diagnosis. Conclusion: These findings may contribute to the development of policies for the identification of breast tumors at an earlier stage. 